In Chapter 2 all concepts of diffraction methods are vividly and concisely described: the Bragg equation, the reciprocal lattice, the structure amplitude, the scattering density and the principles of structure determination. The next step is to discuss the scattering amplitudes in all three usable diffraction methods - X-ray, neutron and electron. After that the peculiarities of each diffraction method branch are treated briefly but all important things are stated clearly.

In this short exposition very valuable remarks are spread here and there about the physical meaningfulness of different techniques so often used somewhat blindly in different structure investigations.

Systematic errors in structure determinations are fully discussed in a special section. Perhaps a half page is lacking to warn the reader of unexpected trouble with which he can be faced if the crystal contains a comparatively small per cent of impurities. I cannot help repeating my opinion that the inner consistency of the structural result is sometimes more important than the $R$ index. The 'clever' structure with $R$ equalling say 0.11 is better than a 'silly' structure with $R=0 \cdot 10$. The importance of introducing structural reasoning as an element of structure determination is, from my point of view, not claimed with sufficient firmness.

Chapter 3 introduces us to optical vibration spectroscopy. Perhaps it would be worth giving a little more place to the Urey-Bradley field. Spectroscopic manifestations of hydrogen bonding are described briefly and I think this is right bearing in mind the book of Pimentel \& McClellan.

The sections dedicated to neutron magnetic resonance methods are to be highly welcomed. At the moment no one method (I ask the optical spectroscopists not to be angry with me) has a comparable value as a partner of the diffraction methods. The n.m.r. method, as is clearly shown in a special section of the book, is well suited to the determination of proton-proton (or F-F etc.) distances. In the next chapter it is shown that without n.m.r. techniques we have no means of investigating the reorientation movements of molecules and this is especially valuable as in many cases even we did not suspect the presence of such movements.

But let us return to Chapter 3. Here we get acquainted with potential functions used for describing hydrogen bonding. It is shown quite clearly that the potentials can be checked with different diffraction and spectroscopic experiments. Some examples of using both techniques are given at the end of Chapter 3. But the importance of the n.m.r. method is insufficiently stressed. One of the n.m.r. applications is buried in a section on diffraction and optical spectroscopy. The other (reorientation problem) is dealt with in the neutron spectroscopy section en passant.

So Chapter 4 is in the first instance an exposition of neutron inelastic scattering. I should be happier if this chapter occupied another 5-10 pages, at the expense of the last part of the book if necessary.

In Chapter 5 we find information about hydrogen-bonded organic and biological molecules; in Chapter 6 are discussed the hydrogen bonds in hydrates and other inorganic crystals. (Why 'other'? There are plenty of organic hydrates.) Chapter 7 is dedicated to the hydrogen bonded ferroelectrics. The last chapter (two pages) has a title - 'Outlook for the future'.

Summing up I can say that the Hamilton-Ibers book is certainly a happy occurrence. The reading of the method- ical parts is surely stimulating. The pages where hydrogenbonded structures are described give the reader a lot of useful information.

The book is illustrated with stereoscopic drawings. It is amusing and instructive to search for a three-dimensional view of the structure.

The references are voluminous. So, from all points of view the book is a good introduction not only to the hydrogen-bond problem but to the methods of crystal structure investigation.

\section{A.I. KITAYGORODSKY}

Institute of Elemento-Organic Compounds (INEOS)

Academy of Sciences of the USSR

Vavilov Street 14

Moscow

USSR

\section{The physics of large deformation of crystalline} solids. Springer tracts in natural philosophy Vol. 14. By J.F. Bell. Pp.viii +253 . Berlin, Heidelberg, New York: Springer Verlag, 1968. Price DM 48, $\$ 12.00$.

From its title, crystallographers might expect this book to deal with crystal lattices and their imperfections; in fact, these are scarcely mentioned. It is actually a review of 'nearly 2000 individual uniaxial stress experiments in 19 crystalline elements and several binary combinations. The experiments include many different force-time histories and metallurgical states'. From these data a generalized large deformation behaviour is demonstrated.

It is shown that all of the nominal stress-strain curves have an equation of the form

$$
\sigma^{2}=\beta^{2}\left(\varepsilon-\varepsilon_{b}\right)
$$

where $\sigma$ and $\varepsilon$ are respectively the stress and strain, and $\varepsilon_{b}$ is the intercept of the parabola on the strain axis. $\beta$ is given by the equation

$$
\beta=\left(\frac{2}{3}\right)^{r / 2} \mu(0) B_{0}\left(1-T / T_{m}\right),
$$

where $\mu(0)$ is the shear modulus of the isotropic material at absolute zero, $T$ the absolute temperature of the experiment, $T_{m}$ the melting point, and $B_{0}$ a universal constant. $r$ is always an integer. Different segments of a given stressstrain curve will have different values of $r$ and $\varepsilon_{b}$, but the strains at which the transitions between segments occur, form a series which is the same for all elements. It is further demonstrated that the value of $\mu(0)$ is always one of the series of values given by

$$
\mu(0)=\left(\frac{2}{3}\right)^{s / 2}\left(\frac{2}{3}\right)^{p / 4} A
$$

where $s$ and $p$ are integers and $A$ is a universal constant. Changes in $s$ and $r$ are shown to be equivalent.

A research monograph of this type should meet two requirements: it should be understandable by the student with only a general background knowledge of the subject, without reference to the numerous papers which it should supersede, and it should convince the reader that it is more than curve fitting.

I feel the book does not fully meet the first requirement since the student would, in several places, be forced to consult the original papers. It is unfortunate that there is an annoying mixture of units, stresses being given some- 
times in p.s.i. and sometimes in $\mathrm{kg} . \mathrm{mm}^{-2}$. The author is rightly at pains to emphasize the enormous amount of experimental data which has been analysed in demonstrating the generalization, but this, at times, gives the impression of reading a catalogue. Original experiment numbers have been retained in labelling curves, which makes it difficult to find on a diagram the particular curve, or group of curves, discussed in the text. Uniformity of units, greater selectivity in presenting data for discussion, and easier reference between text and Figures would improve the readability of the book.

On the second criterion the book nearly succeeds: one is almost convinced that there is more to the generalization than curve fitting. The scope of the experiments is enormous, and the degree of agreement between the data and the generalization is very impressive. The industry and tenacity of the author are admirable and his experimentation brilliant. However, I have one nagging doubt. Curves of $\sigma^{2}$ against $\varepsilon$ are frequently considered, and the generalization can then be demonstrated by showing that straight lines having a discrete sequence of slopes, but an adjustable intercept, can be fitted to the data. Because of the proximity of the transition strains, the number of points to be fitted to any one straight line can be very few. Is the method, then, very critical? Can any smooth, shallow curve be broken down to a series of short, straight lines of given slope? Discussion of these questions might have convinced me completely.

Despite these criticisms I found the book intriguing, asking questions as well as answering them, and making me want to get involved in the subject. This, surely, is the measure of a good research monograph.

\section{Physics Department}

University of Manchester

Institute of Science and Technology

Manchester 1

England

Theory of elastic waves in crystals. By FEDOR $I$. FEDOROV, translated from the Russian by J.E.S. Bradley. Pp. $375+19$ Figs. New York: Plenum Press, 1968. Price $\$ 25$.

The modern developments involving ultrasonic generators and detectors, piezoelectric resonators and phonon interaction with $\mathrm{X}$-rays and other types of radiation all require an understanding of the transmission of elastic waves through solid bodies and especially through crystals. Many standard works on the physical properties of crystals have one or more chapters devoted to the study of the propagation of plane elastic waves through crystals of various symmetries. However, the book under review is remarkable in that it is entirely devoted to this subject. It is based on a series of lectures given to graduate students in Moscow University but it would be misleading to imply that it is a book which students will find easy to read. It has the character of a monograph rather than that of a student text. In a foreword, H.B. Huntington says 'the author has gone to considerable pains to develop in his mathematical background a consistent tensor framework which acts as a unifying motif throughout the various aspects of the subject'. The author also explains in the Preface to the Russian edition that his method is based on general methods of vector and tensor calculus which do not necessitate explicit statement of the tensor components. This generalized tensor analysis makes the reading unfamiliar and, until the language is learned, rather difficult, but it gives a unity to the whole presentation.

There are nine chapters, the first three of which cover the relatively familiar ground leading to Christoffel's equation and its applications. Chapter 4 gives an account of the flow of energy and the form of wave surfaces. In the next three chapters the theory is applied, first to an isotropic medium, and then to hexagonal, cubic, tetragonal and trigonal crystals. Chapter 8 is concerned with the reflexion and refraction of elastic waves from plane boundaries and covers ground not usually to be found in the standard works. The last chapter deals with the calculation of Debye temperatures taking into account the elastic properties of cubic and hexagonal crystals.

The translation appears to be excellent and the production is good except that the suffixes are often rather too small to be seen easily.

\section{Cherry Hinton Road Cambridge \\ England}

W. A. WOOSTER

Anisotropy in single-crystal refractory compounds, Volumes 1 and 2. Edited by FRED W. VAHLDIEK and Stanley A. Mersol. Pp. xix +405 Vol. 1, xi+ 493 Vol. 2. New York: Plenum Press, 1968. Price $\$ 45$ set.

These volumes contain the papers presented at an international symposium sponsored by the Ceramics and Graphite branch of the Materials Laboratory of the United States Air Force, and held at Dayton, Ohio in June 1967. The range of the topics which are covered is far wider than the title of the volumes suggests. All the papers deal with some aspect of the behaviour of refractory compounds, with the exception of one excellent paper on the mechanical properties of beryllium. Subjects which are discussed include the electronic structure of transition metal carbides, the defect structure of non-stoichiometric compounds and problems in the chemical analysis of materials. Mechanical properties are prominent in Volume 2. The papers are in general more expansive and detailed than journal publications and in some cases review results accumulated over a considerable period of time. These points may go some way towards justifying these expensive books.

Department of Metallurgy

G.W. Groves

University of Oxford

Parks Road

Oxford

England

Proceedings of the Symposium on Low Energy Electron Diffraction, Ramada Inn, Tucson, Arizona, February 1968. Transactions of the American Crystallographic Association, Vol. 4. 1968. Pp. v+114. Price $\$ 5.00$ post paid from Polycrystal Book Service, P.O. Box 11567, Pittsburgh, Penn. 15238, U.S.A.

Low energy electron diffraction (LEED) has been the subject of a rapid growth of interest over the last three 